pandemics match the documented symptoms of today's plague. That Y. pestis was the aetiological agent of the third pandemic is irrefutable — the plague bacillus was first isolated and identified by Alexander Yersin during this outbreak. Recent molecular studies with $Y$. pestis provide additional evidence linking the bacterium with the earlier great pandemics. The 'molecular clock' (baseline mutations in housekeeping genes) suggests that $Y$. pestis evolved somewhere between 1,500 and 20,000 years ago, the former figure in remarkable agreement with the appearance of the justinian plague. Additionally, molecular phylogeny has revealed three genetically defined groups of $Y$. pestis, and these appear to correspond to the groups of strains associated with the three pandemics of plague.

It should be possible to obtain conclusive evidence that Y.pestis was the causative agent because a large number of corpses were buried anonymously under towns and cities during the Black Death. Viable bacteria will be long gone, but tell-tale DNA might still be present. But although one research group has reported the isolation of $Y$. pestis DNA from the teeth of presumed plague victims, others have been unable to repeat these studies.

Together these books give a good account of the history of the three great plagues. Other aspects are less convincing. We do not yet have conclusive proof that $Y$. pestis was the cause of the three great plagues. Although I am not yet convinced that the mysterious and unidentified virus suggested by Scott and Duncan was the cause of the Black Death, these books do serve to remind us never to be blinkered to other possibilities.

Richard W. Titball is at the Defence Science and

Technology Laboratory, Porton Down,

Salisbury SP4 0JQ, UK.

\section{Clash of the titans}

\section{Einstein Defiant: Genius versus} Genius in the Quantum Revolution by Edmund Blair Bolles

Joseph Henry Press: 2004. 256 pp. \$27.95

\section{Arthur Fine}

The authors of postmodern novels enjoy the liberty of moving around randomly through time, thereby erasing the appearance of causality and challenging our sense of rational action. In Einstein Defiant, Edmund Bolles also zigs and zags randomly through time, but he is not trying to erase the modalities of human understanding. Rather, he is trying to show how entangled that understanding is with the concrete particulars of time, place and culture.

The geniuses of the subtitle are Albert

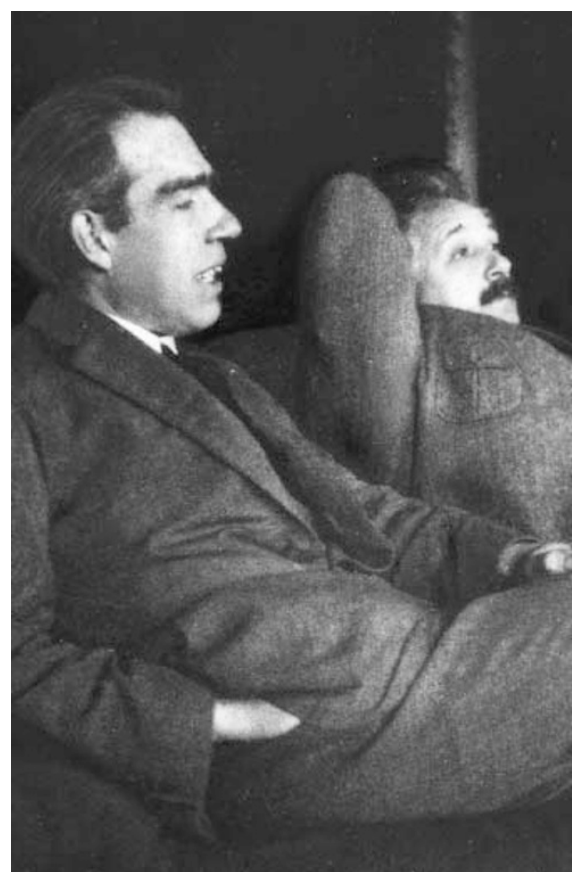

In dispute: Niels Bohr (left) and Albert Einstein.

Einstein and Niels Bohr, and 'defiant' reflects Einstein's reservations about quantum theory. Although Einstein initially responded with enthusiasm to the new physics, this quickly gave way to disappointment. His reservations were twofold. First, he felt that quantum mechanics had abdicated the historical task of science to furnish us with knowledge of nature that is independent of observers or their acts of observation. The role of the wave function in quantum theory was to provide probabilities for 'results' if appropriate measurements were made (the Born rule). The theory was silent about what, if anything, was likely to be true in the absence of observation. In this sense, it was irrealist. Second, quantum mechanics was essentially statistical and, unlike probabilities in classical statistical mechanics, quantum probabilities were not understood as arising from ignorance of fine details. In this sense, the theory was indeterministic.

Einstein began to probe how strongly quantum theory was tied to irrealism and indeterminism, leading to a series of debates over its interpretation, beginning around the Solvay Conference of 1927 and culminating in the so-called EPR paper of 1935 (A. Einstein, B. Podolsky and N. Rosen Phys. Rev. 47, 777-780). That paper introduced quantum entanglement and laid the groundwork not only for John Bell's investigations into nonlocality, but also for the contemporary development of quantum information theory (computing, cryptography and teleportation).

Einstein Defiant lays out the background of these debates in the context of the 'old' quantum theory, whose duality of waves and particles set the agenda for the new quantum mechanics of 1925-27. It continues the story up to the Solvay Conference of 1930. In discussions at the conference, Einstein's 'photon-in-a-box' challenged the energytime uncertainty relation. After a sleepless night, Bohr brought considerations of clock times from general relativity to bear, and saved the day for quantum uncertainty. Bolles uses this triumph as a nice counterpoint to Bohr's earlier defeat over the Bohr-Kramer-Slater theory in which, letting go of strict energy conservation, Bohr had hoped to maintain a wave picture.

By jolting the reader around in spacetime, Bolles' nonlinear history produces vivid impressions of times, places and personalities. Moreover, Bolles manages to describe developments in physics with imaginative analogies and contrasts (wave packets as bulges moving through a bullwhip, or the orbit-jumping electron of the Bohr theory as the ghost of Hamlet's father - it's here, it's there, it's nowhere in between). Although equation-avoidance is standard policy in semi-popular science writing, it frequently fails because one wellplaced equation may be worth more than a thousand words. Nevertheless, the policy works here because Bolles blends his analogies seamlessly into the historical vignettes around which he weaves his story.

Inevitably, some of the analogies that carry the physics are strained but, overall, Bolles stays true to the physics while keeping the images lively (and his readers awake). One spot that does need attention is his account of the uncertainty formulas, where he confuses the operators $p$ and $q$ with the uncertainties $\Delta p$ and $\Delta q$ and overlooks the statistical character of quantum uncertainty.

Of course, it is not only the physics that needs explaining. The philosophical concepts - especially realism and determinism, and Bohr's difficult 'complementarity' — are no more transparent than is the Schrödinger equation. Bolles does an excellent job with probability and determinism - his nuanced discussion of indeterminism in connection with Heisenberg is particularly fine. He fares less well in explaining the issues surrounding realism. Indeed, he makes it appear that Bohr endorsed the idea that measurements actually create the physical attributes that correspond to their outcomes, and that quantum mechanics simply runs counter to the belief that the micro-world of electrons and such is real. As for complementarity, Bolles wisely begs off, noting early on that trying to pin down Bohr is "like trying to cage a beautiful cloud".

All in all, this is a delightful book. It succeeds admirably in capturing the spirit of the people, the times, the science and the intellectual passion that marked the rise of quantum physics.

Arthur Fine is in the Department of Philosophy, University of Washington, Seattle, Washington 98195-3550 USA. 